\title{
Methodology of Precise Skull Model Creation
}

\author{
James Xia ${ }^{1}$, Jaime Gateno ${ }^{1}$, John Teichgraeber ${ }^{2}$, Andrew Rosen $^{3}$ \\ 1. Department of Oral and Maxillofacial Surgery \\ 2. Department of Pediatric Surgery \\ 3. Department of Orthodontics \\ University of Texas Health Science Center at Houston \\ Houston, Texas, U.S.A. \\ \{James.J.Xia, Jaime.Gateno, John.F.Teichgraeber\}@uth.tmc.edu
}

\begin{abstract}
While CT imaging is excellent for demonstrating bone structures, it is unable to display an accurate rendition of the teeth. To address this problem, a method of incorporating accurate teeth into the CT scan was developed. This method combined a 3D CT bone model with digital dental models creating a "precise skull model". An experiment was completed to test the accuracy with which the digital dental models were incorporated into the CT bone models.
\end{abstract}

\section{Introduction}

Computed Tomography (CT) imaging is excellent for generating bone models. However, a significant disadvantage of CT is that it is not capable of accurately representing the teeth. The current maxillofacial surgical planning systems still need to employ conventional dental model surgery to establish the occlusion and fabricate surgical splints. Plaster dental models, mounted on articulators, are the most accurate replicas of the patient's teeth. However, the models themselves lack bony support. The limitation of conventional dental model surgery is that the planner cannot visualize the surrounding bony structures, which is critical in the treatment of complex cranio-maxillofacial deformities.

The purpose of this study was to create a "precise skull model", which accurately represents both bony structures and teeth. The authors developed a technique to create a computer bone model with accurate teeth by incorporating digital dental models into a CT bone model, called "precise skull model". This was done to avoid the need for dental model surgery, or the need for incorporating plaster dental model into a stereolithographic model. The digital dental models were obtained by laser surface scanning dental impressions, and they were incorporated into the CT bone models by using fiducial markers.

\section{Materials and Methods}

The study was completed in three steps. The first step was to create digital dental models. The second step was to incorporate the digital dental models into 3D CT skull 
model, creating a "precise skull model". The final step was to assess the accuracy of this "precise skull model".

\subsection{Creation of Digital Dental Models}

Prior to obtaining dental impressions, fiducial markers were inserted into a radiolucent full-arch dental impression tray (triple-tray, ESPE America, Norristown, PA) (Fig.1). This triple-tray was used to take simultaneous impressions of the maxillary and mandibular arches. Four fiducial markers were mounted on the tray. One pair was at right and left canine region, another pair was at right and left molar region (Fig.2). Dental impressions were then taken in the conventional manner.

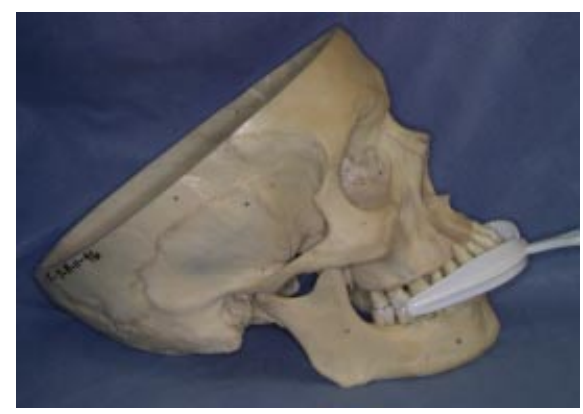

Fig.1 Triple tray

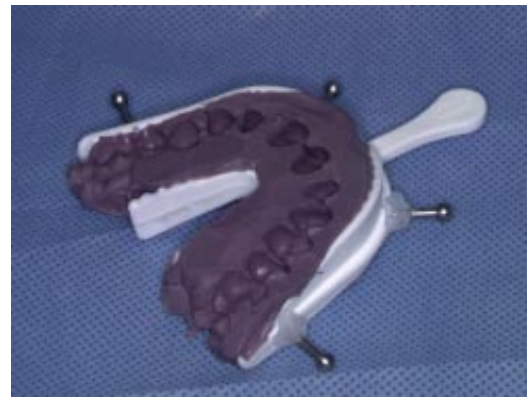

Fig.2 Fiducial markers

The dental impressions with the four fiducial markers were scanned using a 3D laser surface scanner. Using a custom program, the scanned impression was turned inside out to generate from the negative model of the impression a positive model of the teeth. A digital dental model with four fiducial markers was created.

\subsection{Incorporation of Digital Dental Models into a 3D CT Bone Model}

With the same dental impressions and fiducial markers in place, a CT scan was taken at a thickness of $1.0 \mathrm{~mm}$. The digital CT data was directly transferred from the CT scanner to a personal computer using a 5.25" MO disk drive.

A 3D CT skull model with four fiducial markers was reconstructed via Marching Cubes algorithm and the total numbers of triangles were reduced to 210,000 via Decimation algorithm. These fiducial markers were located in the exactly same position as they were on the digital dental models.

Using another custom computer program, interactive alignment of these corresponding fiducial markers was made between the bone model and the digital dental models. After the fiducial markers were aligned, the less than accurate dentition in the 3D skull model was replaced by the accurate dentition of the digital dental models. The fiducial markers were then removed and a "precise skull model" was created (Fig.3). 


\subsection{Assessment of Accuracy of "Precise Skull Model"}

A dry skull with intact dentition was employed. Digital dental models were first created. The dry skull was then CT scanned in order to generate 3D bone model. The digital dental models were incorporated into the $\mathrm{CT}$ bone models to create a "precise skull model".
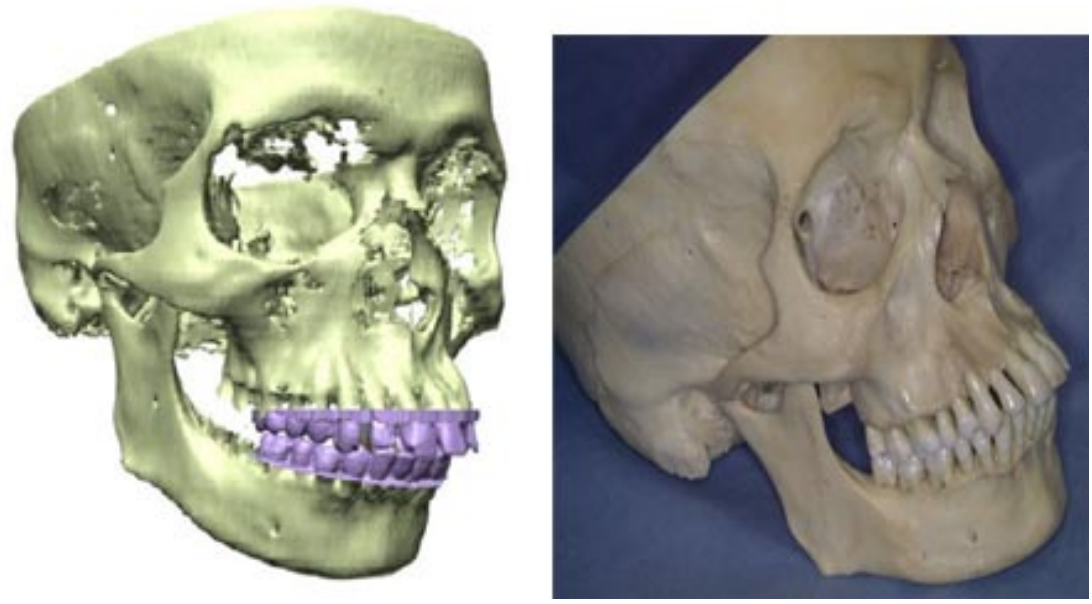

Fig.3 Comparison between "Precise skull model" and dry skull

Measurements were made on the "precise skull model". The measurements were grouped into three categories: bone-to-bone, tooth-to-tooth and bone-to-tooth measurements (Table 1). The bone-to-bone measurements were made between two bony landmarks and were used to assess the accuracy of the $3 \mathrm{D}$ bone model. The tooth-to-tooth measurements were made between two dental landmarks and were used to assess the accuracy of the digital dental models. The bone-to-tooth measurements were made from a bony landmark to a dental landmark and were used to assess the accuracy of the alignment of the digital dental models to the 3D CT bone model. The same measurements were made directly on the dry skull utilizing a Boley gauge. All measurements were performed by the same investigator (A.R.) and each measurement was repeated three times on different days.

The means, standard deviations and variances of the measurements of the 3D model and the dry skull were computed respectively. Person's correlation coefficient of variance was performed to identify any possible differences between the $3 \mathrm{D}$ model and the dry skull.

\section{Results}

A "precise skull model" was created. This computer model not only represented bony structures from CT data, but also represented dentition from digital dental models. 
The intra-observer variances ranged from 0.00 to 0.42 on "composite skull model" and from 0.00 to 0.09 on dry skull. There were no significant differences among the three measurements made by the investigator. Therefore, the three measurements were averaged for each item.

The mean differences between the computerized "precise skull model" and the dry skull were also calculated. Their means and standard deviations were then computed. For the bone-to-bone measurements, the average difference was $0.50 \mathrm{~mm} \pm$ $0.62 \mathrm{~mm}$. For the tooth-to-tooth measurements, the average difference was $-0.06 \mathrm{~mm} \pm$ $0.19 \mathrm{~mm}$. For the bone-to-tooth measurements, the average difference was $0.23 \mathrm{~mm} \pm$ $0.30 \mathrm{~mm}$. The average difference for all measurements was $0.24 \mathrm{~mm} \pm 0.48 \mathrm{~mm}$ (Table 2).

The Pearson's correlation coefficient of variance was calculated for each group of measurements. It was 0.9997 for the bone-to-bone measurements, 0.9999 for the bone-to-tooth measurements, and 0.9999 for the teeth-to-teeth measurements. There is no statistical significant difference between the 3D skull model and the dry skull.

\section{Discussion}

Three-dimensional CT modeling for diagnosis and treatment planning in orthognathic surgery has demonstrated significant potential. ${ }^{1}$ It has the ability to simplify surgical procedures, decrease anesthesia time, and increase the accuracy of the intended surgical outcomes.

Three-dimensional CT bone model is excellent at representing bony structures, but it is only an approximation of the patient's actual structures. The precision of a 3D CT model is limited by the layer thickness during CT scanning. CT scanners are only able to capture images layer by layer, data between the image layers is reconstructed by mathematic algorithms, i.e. Marching Cubes. Interocclusal relationship of maxillary and mandibular arches plays a major role as reference and is the key to an excellent surgical outcome. However, the 3D CT model is not accurate enough to reproduce interocclusal relationships because of the complexities of dental anatomy. Furthermore, it is not possible to remove scattering, which was caused by metal orthodontic brackets, dental filling or prosthesis during CT scanning. The current surgical planning systems still need to employ conventional dental model surgery to establish the occlusion and fabricate surgical splints.

Plaster dental models, mounted on articulators, are the most accurate replicas of the patients' teeth. However, the models themselves lack bony support. The surgical plan for a plaster dental model is only based on the physical examination, radiography and photography. The limitation of this approach is that the planner cannot visualize the surrounding bony structures, which are critical in the treatment of complex maxillofacial deformities. Stand-alone digital dental models, generated by a laser scanner, have the same shortcoming as plaster dental models. 
Several investigators have attempted to incorporate dental models into a CT bone model. ${ }^{[6,7]}$ In order to accomplish this, they developed different methods for replacing the teeth in 3D stereolithographic models with plaster dental models. The existing dentition in the stereolithographic model was removed and plaster dental models were physically inserted. A specially designed face-bow was used to align the plaster dental models to the stereolithographic model. However, these methods were based on physical models, which were not suitable for virtual osteotomies.

The authors have created a three-dimensional "precise skull model" for orthognathic surgical planning. This computerized model precisely represents both bony structures and dentition. By using the authors' method, digital dental models with accurate occlusal relationships can be directly incorporate into a 3D CT bone model, traditional plaster dental model surgery will be unnecessary and totally replaced by computerized virtual osteotomies. The accuracy of the "precise skull model" was demonstrated by Pearson's correlation coefficient. The tooth-to-tooth and the bone-to-tooth measurements demonstrated a high degree of accuracy in the digital dental models and their incorporation into 3D CT bone model. There was only a small degree of variance in bone-to-bone measurements $(0.5 \mathrm{~mm} \pm 0.6 \mathrm{~mm})$ between the computerized "composite skull model" and the dry skull, which was due to the limitation of current CT technology. The accuracy of this precise skull model is also superior to other methods. ${ }^{[2-7]}$ Terai et al published an error of $4.2 \mathrm{~mm}$ while incorporating plaster dental models into a 3D stereolithographic model. ${ }^{[7]}$

This "precise skull model" can be used for accurate computer diagnosis and treatment planning. It also can be used to generate a stereolithographic model of the patient's craniofacial skeleton and dentition. The next logical step is to determine whether surgical splints can be fabricated from this "precise skull model".

\section{References}

1. Bill J Reuther JF, Betz T, Dittmann W and Wittenberg G. Rapid prototyping in head and neck surgery planning. J Cranio Maxillofac Surg. 24:20-1, 1996

2. Karcher H. Three-dimensional craniofacial surgery: transfer from a three-dimensional (Endoplan) to clinical surgery: a new technique (Graz). J Cranio Maxillofac Surg. 20:12531,1992

3. Lambrecht JT. 3D modeling technology in oral and maxillofacial surgery. Quintessence: Chicago, IL. Pp61-4, 1995

4. Okumura H, Chen LH, Yokoe Y, Tsutsumi S, Oka M. CAD/CAM fabrication of occlusal splints for orthognathic surgery. J Clin Orthodont. 23:231-5, 1999

5. Rose EH, Norris MS, Rosen JM. Application of high-tech three-dimensional imaging and computer-generated models in complex facial reconstructions with vascularized bone grafts. Plast Reconstr Surg. 91:252-64, 1993

6. Santler G. The Graz hemisphere splint: a new precise, non-invasive method of replacing the dental arch of 3D-models by plaster models. J Cranio Maxillofac Surg. 27:169-73, 1999

7. Terai H, Shimahara M, Sakinaka Y, Tajima S. Accuracy of integration of dental cases in three-dimensional models. J Oral Maxillofac Surg. 57:662-5, 1999 


\begin{tabular}{|c|c|c|}
\hline GROUP & LANDMARKS & DEFINITIONS \\
\hline \multirow{10}{*}{$\begin{array}{c}\text { BONE TO } \\
\text { BONE }\end{array}$} & $\mathrm{R}$ Po-Me & Right porion to menton \\
\hline & L Po-Me & Left porion to menton \\
\hline & L Go-Me & Left gonion to menton \\
\hline & R Go-Me & Right gonion to menton \\
\hline & Go-Go & Right gonion to left gonion \\
\hline & $\mathrm{Zy}-\mathrm{Zy}$ & Right zygomatic arch to left zygomatic arch, smallest distance \\
\hline & L Po-L Or & Left porion to left orbitale \\
\hline & R Po-R Or & Right porion to right orbitale \\
\hline & Max width & Smallest width of maxilla at Lefort 1 level \\
\hline & Man width & Smallest width of mandible, ramus to ramus \\
\hline \multirow{8}{*}{$\begin{array}{c}\text { тоОтн то } \\
\text { тоОтн }\end{array}$} & U3-U3 & Upper right cuspid to upper left cuspid, buccal surfaces \\
\hline & L3-L3 & Lower right cuspid to lower left cuspid, buccal surfaces \\
\hline & U6-U6 & Right upper first molar to left upper first molar, buccal surfaces \\
\hline & LL6-LR3 & Lower left first molar to lower right cuspid, buccal surfaces \\
\hline & LR6-LL3 & Lower right first molar to lower left cuspid, buccal surfaces \\
\hline & UR6-UL3 & Upper right first molar to upper left cuspid, buccal surfaces \\
\hline & UL6-UR3 & Upper left first molar to upper right cuspid, buccal surfaces \\
\hline & $\mathrm{U} 2-\mathrm{U} 2$ & Upper right lateral to upper left lateral, distal surfaces \\
\hline \multirow{8}{*}{$\begin{array}{c}\text { BONE TO } \\
\text { TOOTH }\end{array}$} & RL3-Me & Right lower cuspid tip to menton \\
\hline & LL3-Me & Left lower cuspid tip to menton \\
\hline & LU3-L Or & Upper left cuspid tip to left orbitale \\
\hline & RU3-R Or & Upper right cuspid tip to right orbitale \\
\hline & Na-RU3 & Nasion to upper right cuspid \\
\hline & Na-LU3 & Nasion to upper left cuspid \\
\hline & Na-RU6 & Nasion to upper right first molar \\
\hline & Na-LU6 & Nasion to upper left first molar \\
\hline
\end{tabular}

Table 1 Definitions of measurement landmarks by group 


\begin{tabular}{|c|c|c|c|c|}
\hline GROUP & LANDMARK & $\begin{array}{c}\text { 3D MODEL } \\
\text { Average }\end{array}$ & $\begin{array}{c}\text { SKULL } \\
\text { Average }\end{array}$ & Difference \\
\hline \multirow{10}{*}{ BONE TO BONE } & R Po-Me & 129.30 & 129.62 & 0.32 \\
\hline & L Po-Me & 127.81 & 129.15 & 1.34 \\
\hline & L Go-Me & 80.04 & 81.54 & 1.50 \\
\hline & R Go-Me & 79.54 & 78.87 & -0.67 \\
\hline & Go-Go & 95.03 & 95.79 & 0.76 \\
\hline & $Z y-Z y$ & 120.86 & 121.51 & 0.65 \\
\hline & R Po-R Or & 80.21 & 80.54 & 0.33 \\
\hline & L Po-L Or & 80.39 & 80.58 & 0.19 \\
\hline & Max width & 66.38 & 66.66 & 0.28 \\
\hline & Man width & 95.49 & 95.75 & 0.26 \\
\hline \multicolumn{4}{|c|}{ Average difference } & 0.50 \\
\hline \multicolumn{4}{|c|}{ SD } & 0.62 \\
\hline \multirow{8}{*}{$\begin{array}{l}\text { тоОтн то } \\
\text { тоОтн }\end{array}$} & U3-U3 & 43.71 & 43.78 & 0.07 \\
\hline & U6-U6 & 60.81 & 60.90 & 0.09 \\
\hline & L3-L3 & 32.13 & 32.04 & -0.09 \\
\hline & LL6-LR3 & 49.91 & 49.49 & -0.42 \\
\hline & LR6-LL3 & 50.53 & 50.66 & 0.13 \\
\hline & UR6-UL3 & 57.73 & 57.59 & -0.14 \\
\hline & UL6-UR3 & 58.75 & 58.52 & -0.23 \\
\hline & U2-U2 & 34.54 & 34.63 & 0.09 \\
\hline \multicolumn{4}{|c|}{ Average difference } & -0.06 \\
\hline \multicolumn{4}{|c|}{ SD } & 0.19 \\
\hline \multirow{8}{*}{$\begin{array}{c}\text { BONE TO } \\
\text { TOOTH }\end{array}$} & RU3-R Or & 53.92 & 54.59 & 0.67 \\
\hline & LU3-L Or & 55.10 & 55.10 & 0.00 \\
\hline & RL3-Me & 46.83 & 47.04 & 0.21 \\
\hline & LL3-Me & 46.72 & 47.42 & 0.70 \\
\hline & $\mathrm{Na}-\mathrm{RU} 3$ & 85.69 & 85.70 & 0.01 \\
\hline & Na-LU3 & 85.93 & 86.02 & 0.09 \\
\hline & Na-RU6 & 86.30 & 86.25 & -0.05 \\
\hline & $\mathrm{Na}-\mathrm{LU} 6$ & 87.03 & 87.28 & 0.25 \\
\hline \multicolumn{4}{|c|}{ Average difference } & 0.23 \\
\hline \multicolumn{4}{|c|}{ SD } & 0.30 \\
\hline \multicolumn{4}{|c|}{ Average difference for all groups } & 0.24 \\
\hline \multicolumn{4}{|c|}{ SD for all groups } & 0.48 \\
\hline
\end{tabular}

Table 2 Comparison of "precise skull model" and dry skull 